\section{Técnicos en enfermería en Chile: mirada a su formación desde las competencias declaradas en los perfiles de egreso}

\author{
FRANCISCA VALDEBENITO-ACOSTA ${ }^{1, a}$, \\ JULIO HASBÚN-MANCILLA ${ }^{1, \mathrm{~b}}$, EDUARDO SANTANDER ${ }^{1, \mathrm{c}}$, \\ PIERINNA GALLARDO $2, \mathrm{~d}$
}

\section{Competence profiles for technicians in higher-level nursing, declared by different teaching institutions}

Background: The career of Technician in Higher-Level Nursing (TENS in Spanish) is the technical career that has the higher number of students in Chile. There are important disparities among the higher education institutions that teach it. Aim: To analyze differences in competencies declared in TENS career graduate profiles by type of higher education institution. Material and Methods: Using Atlas.ti 7, we carried out a content analysis of 31 graduate profiles of TENS, published in websites of Universities, Professional Institutes and Technical Training Centers that teach the career, during 2019. Results: Procedural competencies are predominant in graduate profiles, followed by interpersonal ones, with cognitive and ethics competencies being the least declared. Accredited higher education institutions consistently declare more cognitive and ethics competencies than those that are not accredited. Conclusions: There is a procedural bias in graduate profiles, which tends to under declare cognitive and ethics competencies, fundamental for decision-making processes.

(Rev Med Chile 2021; 149: 829-835)

Key words: Chile; Competency-Based Education; Education; Nursing Assistants; Professional Competence.

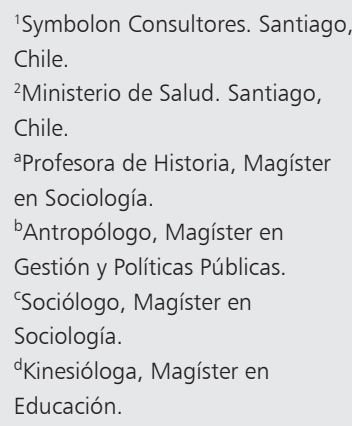

Financiamiento: Esta

investigación fue realizada en e contexto del estudio "Análisis de contenido de los perfiles de egreso de carreras acreditadas en salud", licitación pública 757-126-LE19, solicitada por la Subsecretaría de Salud Pública, Ministerio de Salud.

Los autores declaran no tener conflictos de interés.

Recibido el 2 de octubre de 2020, aceptado el 26 de marzo de 2021.

Correspondencia a:

Francisca Valdebenito-Acosta fvaldebenito@symbolon.cl
$\mathrm{E}$ $\mathrm{n}$ las últimas dos décadas ha habido un incremento sustancial en el acceso a educación superior en Chile ${ }^{1}$. Los datos reportan que la tasa bruta de matrícula en la educación superior en Chile creció de 39,8\% el 2009 a 55\% el 2017, el que se ha producido particularmente en los estudiantes de estratos socioeconómicos más bajos. En efecto, si en el año 1990, la cobertura neta de educación superior de jóvenes de entre 18 y 19 años pertenecientes a $50 \%$ más pobre del país era de $10,5 \%$, el año 2017 sube a $39,1 \%^{2}$.

Información del Ministerio de Educación (MINEDUC) muestra que, considerando el conjunto de las profesiones de salud, la matrícula total pasó de 173.575 estudiantes el año 2010 a 236.617 el año 2019 , constituyendo un incremento de $36,32 \%$. Si se observan las estadísticas totales de matrícula nacional, la carrera de Técnico en Enfermería de Nivel Superior (TENS) tuvo, al año 2019, un total de 44.197 estudiantes matriculados, constituyendo la carrera técnica con más estudiantes en Chile y la segunda carrera más grande en todo el sistema de educación superior ${ }^{3}$. Una razón que contribuye a esta cifra es que la carrera de TENS puede impartirse en los tres tipos de instituciones de educación superior chilenas (IES): Centros de Formación Técnica (CFT), Institutos Profesionales (IP) y Universidades. 
Bajo la categoría TENS hemos reunido las distintas nominaciones dadas por las instituciones de educación superior analizadas, a saber: Técnico en Enfermería, Técnico de Nivel Superior en Enfermería, Técnico Universitario en Enfermería, Técnico en Enfermería (con mención), Técnico de Nivel Superior en Enfermería y Telemedicina, Técnico en Enfermería, Técnico de Nivel Superior en Enfermería, Técnico Universitario en Enfermería, Técnico en Enfermería (con mención), Técnico de Nivel Superior en Enfermería y Telemedicina.

Según una definición provista por los tres colegios profesionales que los agrupan en nuestro país, el TENS "Contribuye y participa como personal de apoyo en acciones de promoción, prevención, diagnóstico, tratamiento y rehabilitación del individuo, familia y comunidad, durante todo el ciclo vital, ejecutando procedimientos de enfermería, participando y colaborando activamente en el equipo de salud y en el cuidado del paciente, de acuerdo con políticas, programas normas y protocolos vigentes con la debida supervisión"4.

El MINEDUC ${ }^{3}$ (2019) reporta que el promedio de años de acreditación de las IES que imparten TENS es de 2,1 años en los CFT, 2,2 años en los IP y 3,3 años en Universidades; respecto a la empleabilidad promedio al primer año se observa que en los CFT es de 43,6\%, en los IP es de 47,0\% y en las Universidades es de $41,1 \%$. Al segundo año luego del egreso se observa que la empleabilidad alcanza 67,2\% en al caso de los CFT, 63,2\% en los IP y un 57,5 en las Universidades. Además, en cada una de las tres IES el ingreso promedio mensual al cuarto año de su egreso fluctúa de $\$ 500.000$ a $\$ 600.000$ pesos. Finalmente, respecto al número de titulados, los CFT aportaron 5.004, los IP 6.389 y las Universidades 592. A partir de los antecedentes anteriores se puede señalar que en general, los IP son las IES que tienen más titulados, junto a los CFT poseen una mejor empleabilidad, y el ingreso promedio es independiente del tipo de IES del cual se egresa.

El campo ocupacional de los TENS se caracteriza por su amplitud, pasando por todos los niveles de la atención (primaria, secundaria y terciaria), en instituciones públicas como privadas, y tanto en el ámbito urbano, como en el rural, por ejemplo, en ocasiones a cargo de las Estaciones Médico Rurales.

La altísima participación de la carrera de TENS en la matrícula nacional de educación superior, y la amplia cobertura de su desarrollo profesional, no han estado aparejadas con un reconocimiento de su importancia en el escenario de las políticas de salud pública en el país. En efecto, hoy el Código Sanitario sólo considera como profesionales del área de la salud a las enfermeras, médicos, odontólogos y químicos farmacéuticos, dejando fuera, por ejemplo, a kinesiólogos, nutricionistas, y tecnólogos médicos, reconociendo a los técnicos de enfermería dentro de las profesiones auxiliares, donde la Contraloría General de la República ha instruido homologar a los TENS como auxiliares paramédicos o afín para el registro de prestadores individuales de la Superintendencia de Salud. Esta situación presenta un vacío regulatorio en términos de la definición de los ámbitos específicos de ejercicio profesional de las distintas profesiones de la salud en los equipos sanitarios y asistenciales ${ }^{5}$. Este contexto ha permitido, por ejemplo, que hoy en día la reglamentación sanitaria vigente no distinga entre los tipos de funciones operacionales de un auxiliar paramédico y de un TENS ${ }^{6}$, lo cual no es consistente con la cantidad y calidad de formación que exhiben: 2 semestres versus 5 semestres; la primera en instituciones reconocidas por las Secretarías Regionales Ministeriales de Salud, la segunda por instituciones de educación superior reconocidas por MINEDUC y la Comisión Nacional de Acreditación.

En este marco, se hace necesario problematizar cuáles son los enfoques formativos que subyacen a las carreras técnicas de salud en Chile, que cristalizan en los perfiles de egreso de las distintas mallas curriculares. En este sentido, es esperable que este contexto de crecimiento vertiginoso se manifieste en una heterogeneidad en la información declarada en los perfiles de egreso.

La presente investigación tiene por objetivo indagar en los perfiles de egreso sintéticos de las carreras de TENS en Chile, buscando aportar evidencia que interrogue críticamente las competencias declaradas por tipo de instituciones de educación superior.

\section{Material y Método}

Investigación cualitativa de carácter exploratorio-descriptivo, mediante análisis de contenido a textos de los perfiles de egreso sintéticos de las páginas web de la carrera de TENS impartidas en 
Chile durante el año 2019 y publicadas en el portal del Ministerio de Educación ${ }^{3}$ "Mi Futuro". Para efectos de comparabilidad entre las distintas IES, fueron incluidos los perfiles sintéticos, es decir, aquellos que ocupaban un máximo de una página, siendo éstos los mayoritariamente publicados por las IES, excluyendo los extensos.

Así, se obtuvo un corpus de 31 perfiles de egreso, asociados a seis tipos de IES en el país, correspondiente a todas las que actualmente imparten la carrera:

Universidades acreditadas $(n=6)$; Universidad Adventista de Chile, Universidad Arturo Prat, Universidad Católica de la Santísima Concepción, Universidad de las Américas, Universidad de Magallanes, Universidad de la Frontera.

Universidades no acreditadas $(\mathrm{n}=1)$; Universidad de Aconcagua.

Institutos Profesionales acreditados $(\mathrm{n}=6)$; IP Dr. Virginio Gómez G, IP Galdámez, IP IPLACEX, IP AIEP, IP de Chile, DUOC UC.

Institutos Profesionales no acreditados $(\mathrm{n}=2)$; IP Diego Portales, IP Valle Central.

Centro de Formación Técnica acreditados $(\mathrm{n}=9)$; CFT IPROSEC, CFT de Tarapacá, CFT Teodoro Wickel Kluwen, CFT Pontificia Universidad Católica de Valparaíso, CFT de la Universidad Católica del Norte, CFT San Agustín, CFT Santo Tomás, CFT de ENAC, CFT INACAP.

Centro de Formación Técnica no acreditados $(\mathrm{n}=7)$; CFT Andrés Bello, CFT ICEL, CFT Instituto Superior de Estudios Jurídicos CANON, CFT Instituto Tecnológico de Chile - I.T.C, CFT Los Lagos, CFT Massachusetts, CFT PRODATA.

Sobre éstos se realizó un análisis de contenido "ciego", vale decir las unidades de estudio fueron codificadas sin conocer la institución de educación superior a la cual pertenecen, mediante un proceso de codificación abierta ${ }^{7}$, utilizando el software Atlas.ti 7.0. Los códigos corresponden al tipo o tipos de saberes curriculares a los que tributan los perfiles de egreso, basándose en la tipología de competencias procedimentales, interpersonales y cognitivas $^{8}$. Las competencias procedimentales son habilidades profesionales asociadas al cumplimiento de normas, protocolos y guías. Las competencias cognitivas por su parte, son saberes genéricos relativos al por qué ocurre un fenómeno. Las interpersonales, son actitudes respecto al saber convivir. A esta tipología se le adicionaron las competencias valóricas, especificándolas de las interpersonales, donde suelen estar ubicadas, a fin de obtener una mayor definición. Éstas se orientan a valores éticos y al rol social que desempeñan los técnicos en enfermería.

\section{Resultados}

La Tabla 1 muestra las competencias declaradas con más frecuencia en los perfiles de egreso sintéticos. En términos generales el número de competencias por dimensión son: procedimental $(n=98)$, interpersonal $(n=50)$, cognitiva $(n=37)$ y valórica $(\mathrm{n}=35)$.

Las competencias más frecuentes declaradas son: en la dimensión procedimental, el apoyo a la promoción, prevención, recuperación, y rehabilitación $(\mathrm{n}=29)$, y la atención básica en enfermería $(\mathrm{n}=28)$; en la dimensión interpersonal, la colaboración en el equipo de atención y/o trabajo en equipo $(n=30)$ y el buen trato $(n=14)$; en la dimensión cognitiva, la resolución de problemas $(n=15)$ y el enfoque biopsicosocial/entorno familia/persona/comunidad/modelo de salud integral $(\mathrm{n}=11)$; finalmente en la dimensión valórica se halló la ética/bioética $(\mathrm{n}=21)$ y el compromiso social $(\mathrm{n}=14)$.

Las competencias con menor frecuencia, pero con menciones mayores o iguales a 5 son: en la dimensión procedimental, la actuación según la normativa vigente o aseguramiento de la calidad de la atención $(\mathrm{n}=13)$, las funciones administrativas $(n=7)$ y el manejo de insumos clínicos $(n=5)$; en la dimensión interpersonal, la comunicación oral y escrita $(n=5)$; en la dimensión cognitiva, el conocimiento teórico practico $(\mathrm{n}=6)$ y la actualización del conocimiento $(n=5)$; no emergiendo menciones en la dimensión valórica.

En síntesis, la Tabla 1 presenta la preponderancia de las competencias procedimentales en la carrera de TENS. También es relevante señalar que 10 instituciones no declaran a la ética/bioética en sus perfiles de egreso sintéticos.

La Tabla 2 muestra las diferencias en las competencias declaradas en el perfil de egreso sintético por tipo de Institución de Educación Superior (IES) y acreditación. En términos generales el promedio de las competencias declaradas por las IES acreditadas es mayor al promedio de las IES no acreditadas.

En promedio, las universidades acreditadas 
Tabla 1. Categorías y subcategorías de análisis de acuerdo a número de menciones en los perfiles de egreso sintéticos $(n=31)$

\begin{tabular}{|c|c|}
\hline Categoría & Sub Categoría \\
\hline \multirow{5}{*}{$\begin{array}{l}\text { Dimensión procedimental } \\
\text { (98 menciones) }\end{array}$} & Promoción, prevención, recuperación y rehabilitación $(n=29)$ \\
\hline & Atención básica de enfermería (n = 28) \\
\hline & Actuación de acuerdo a normativa o aseguramiento de la calidad de la atención ( $n=13$ ) \\
\hline & Funciones administrativas $(\mathrm{n}=7)$ \\
\hline & Manejo de equipos e insumos clínicos $(n=5)$ \\
\hline \multirow{3}{*}{$\begin{array}{l}\text { Dimensión interpersonal } \\
\text { (50 menciones) }\end{array}$} & Colaboración en equipos de salud/trabajo en equipo $(n=30)$ \\
\hline & Buen trato $(n=14)$ \\
\hline & Comunicación oral y escrita $(n=5)$ \\
\hline \multirow{4}{*}{$\begin{array}{l}\text { Dimensión cognitiva } \\
\text { (37 menciones) }\end{array}$} & Resolución de problemas ( $\mathrm{n}=15$ ) \\
\hline & Enfoque biopsicosocial o Entorno persona/familia/comunidad Modelo de salud integral $(n=11)$ \\
\hline & Conocimiento teórico-práctico $(n=6)$ \\
\hline & Actualización del conocimiento $(n=5)$ \\
\hline \multirow{2}{*}{$\begin{array}{l}\text { Dimensión valórica } \\
\text { (35 menciones) }\end{array}$} & Ética-bioética $(n=21)$ \\
\hline & Compromiso Social $(n=14)$ \\
\hline
\end{tabular}

Fuente: elaboración propia. Se incluyen sub categorías con $n \geq 5$.

declaran 7 competencias versus las 3 de su par no acreditada; los Institutos Profesionales acreditados declaran en promedio 5,8 competencias versus 4 de los no acreditados, finalmente los Centros de Formación Técnica declaran en promedio 5,6 competencias versus las 4,7 de los no acreditados.

No se observan diferencias en las competencias procedimentales básicas declaradas, independiente del tipo de IES y acreditación. Sin embargo, sí hallamos diferencias en la dimensión valórica, donde los CFT declaran menos competencias en promedio que las universidades y los institutos profesionales.

En síntesis, la Tabla 2 muestra que no hay diferencias por IES en las competencias centrales de la dimensión procedimental. El promedio de las competencias declaradas por las IES acreditadas es mayor al promedio de las IES no acreditadas. Lo anterior se explica por el mayor número de menciones de competencias en las dimensiones cognitiva y valórica por parte de las IES acreditadas.

\section{Discusión}

El sesgo "procedimentalista" en los perfiles de egreso revisados puede conducir a profundizar las fallas sistémicas del área de la salud, al convertirse en meros gestores de tecnología, exacerbando las dificultades para servir a las comunidades con mayores necesidades 9 . Esto es clave, dado que estos perfiles no deben limitarse a un simple know how, es decir, a una operatoria automatizada y burocrática que excluya la reflexión, el discernimiento ético y los procesos de actualización del conocimiento del futuro TENS ${ }^{10}$.

La competencia para la atención básica y cuidado en enfermería se refiere a una serie de acciones y procedimientos que no son desarrollados en forma aislada de contextos y criterios. Es decir, la distinción entre tipos de competencias es analítica, dado que en la praxis del TENS éstas se conjugan entre sí. Por ello, en las competencias centrales declaradas en los perfiles de egreso de la carrera, 


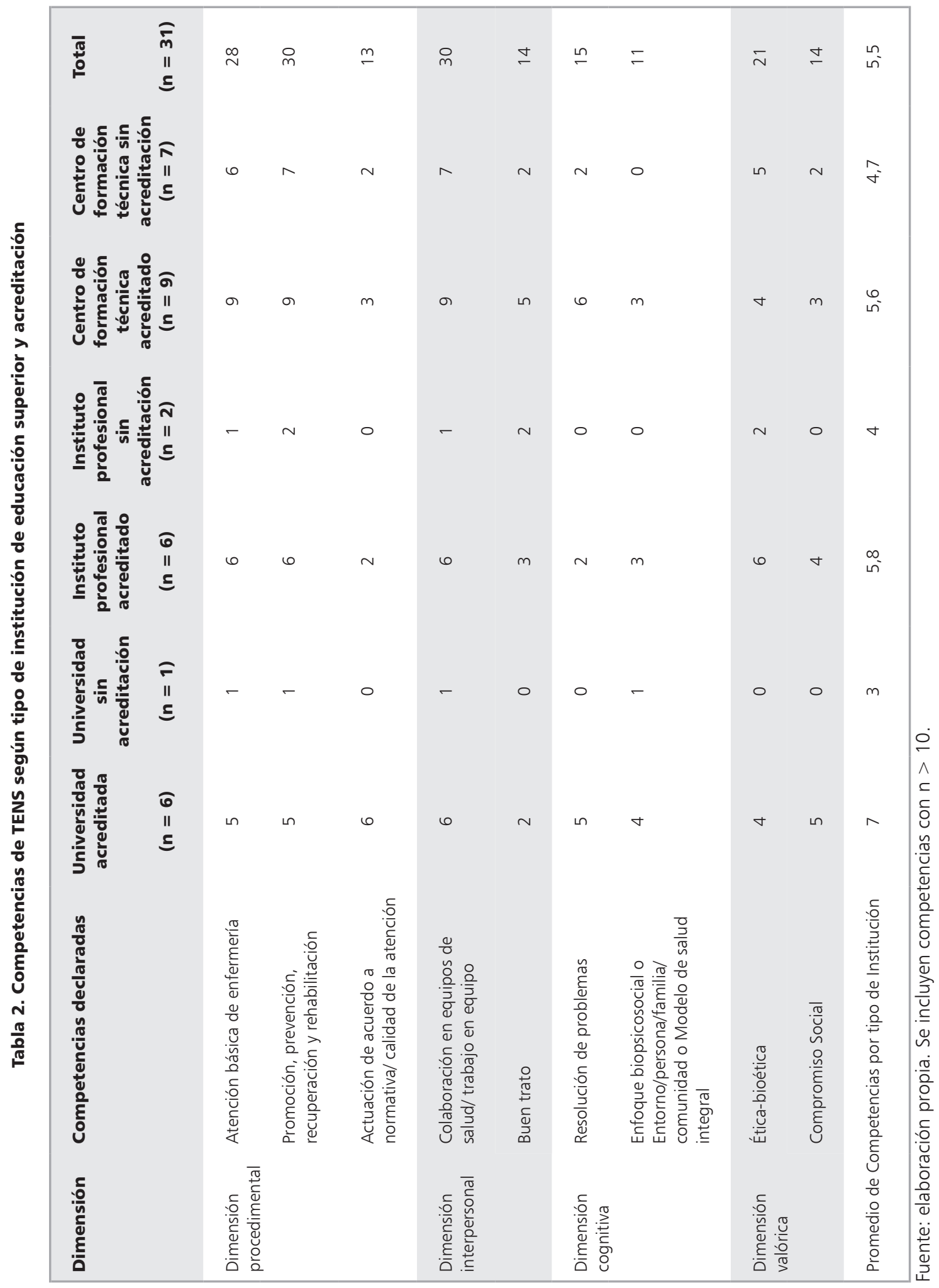


a saber, el apoyo a la promoción, prevención recuperación y rehabilitación, así como la atención básica de enfermería, se espera que los estudiantes pueden movilizar e integrar durante su proceso formativo conocimientos y valores para resolver problemas y guiar su conducta como apoyo al equipo de salud. Esta labor de cuidado requiere por lo tanto de conocimientos, habilidades y actitudes que derivan de un proceso formativo basado no solo en una operatoria procedimental, o bien sus capacidades para conformarse como miembro del equipo de salud, sino en competencias cognitivas y valóricas, que le permitan contar con una guía y método para resolver problemas que incluso pueden exceder el ámbito clínico. Así, el personal técnico de enfermería debe poseer los conocimientos necesarios del situ, es él quien debe asegurar su eficiencia.

Los perfiles de egreso sintéticos analizados se diferencian en forma sustantiva por IES acreditadas y no acreditadas, en los ámbitos cognitivos y valóricos, de acuerdo a las competencias declaradas. Esto es consistente con la literatura que señala que la acreditación es un mecanismo que cumple un papel de control frente a la necesidad de atestiguar la garantía pública de calidad de una institución o programa, en contextos complejos de internacionalización y enorme diversificación ${ }^{11}$.

Las competencias cognitivas y valóricas fueron las menos frecuentes en los perfiles de egreso examinados. Estas últimas resultan fundamentales para desarrollar el juicio técnico en situaciones complejas y emergentes. Su baja presencia puede estar asociada a dos supuestos acerca del rol de los TENS que podrían estar implícitos en la estructura de funcionamiento del equipo de salud: (i) que su acción se encuentra supervisada por un profesional de la salud durante el ejercicio de sus funciones, y (ii) que los protocolos de salud actúan restringiendo la discrecionalidad en las decisiones por parte de los TENS. Sin embargo, los contextos en que se insertan las y los TENS son diversos, desde postas rurales en zonas aisladas hasta hospitales de alta complejidad. Por ende, se requiere que en su ejercicio técnico de apoyo sean capaces de resolver en forma competente los conflictos que se le presentan, por lo que es clave que los perfiles de egreso contemplen un mejor balance hacia componentes de las dimensiones cognitivas y valóricas.

Es especialmente preocupante que un tercio de las IES no declaren competencias de ética/bioética en sus perfiles de egreso sintéticos, ya que están expuestos a enfrentar conflictos de carácter ético debido a las responsabilidades propias asociadas al cuidado de las personas y al hecho de trabajar en un ámbito de salud cada vez más tecnificado y complejo ${ }^{12}$.

Este estudio está limitado por un importante vacío de conocimiento en la literatura respecto a los técnicos en enfermería. Pese a ello, y dada la presencia transversal de los TENS en el Sistema de Salud en Chile, este estudio presenta evidencia inédita en relación a la preeminencia de los procesos de acreditación de las IES que los forman.

Finalmente, en un escenario óptimo para la formación de TENS, debiésemos hallar un balance entre: (i) las necesidades de la población, (ii) la demanda del sistema de salud por técnicos de enfermería, y (iii) la oferta de los mismos por parte del sistema educativo. Idealmente, las IES determinan cuántos profesionales se producen en respuesta a las señales del mercado de trabajo generadas por las instituciones sanitarias, y estas señales deben responder correctamente a las necesidades de la población ${ }^{9}$. Por ello, cuando el escenario funciona alejado de este óptimo, un mecanismo como la acreditación permite asegurar ciertos mínimos, que incluso pueden ser detectados, al menos declarativamente, en los perfiles de egreso sintéticos de las IES.

\section{Referencias}

1. OECD. Educación en Chile, Revisión de Políticas Nacionales de Educación, OECD Publishing, Paris/ Fundación SM, Ciudad de México. 2018. https://doi. org/10.1787/9789264288720-es.

2. Arzola M. Gratuidad y financiamiento de la educación superior: cifras para el debate. Serie Informe Social 174, Libertad y Desarrollo. 2019. Disponible en línea en: https://lyd.org/wp-content/uploads/2019/05/siso-174-gratuidad-y-financiamiento-de-la-educacion-superior-cifras-para-el-debate-febrero2019.pdf. [Consultado el 13 de julio de 2020].

3. MINEDUC. Portal en línea de información a futuros estudiantes de educación superior Mi Futuro. 2019. www.mifuturo.cl [Consultado el 12 de julio de 2020].

4. Montecinos, A. (2020). Historia del TENS. Colegio Nacional de TENS (CONATENS). Disponible en línea en: https://conatens.cl/publicaciones/ 
5. Colegio de Psicólogos. Modificación Código Sanitario Profesiones del Área de la Salud. 2017. Disponible en línea en: http://colegiopsicologos.cl/2017/10/03/modificacion-codigo-sanitario-profesiones-del-area-de-la-salud-presentacion-proyecto-de-ley/. [Consultado el 13 de julio de 2020].

6. González T. Técnicos sin reconocimiento legal: los TENS exigen su espacio. Radio Universidad de Chile. 2019. Disponible en línea en: https://radio.uchile.cl/2019/02/09/ tecnicos-sin-reconocimiento-legal-los-tens-exigen-su-espacio/. [Consultado el 12 de julio de 2020].

7. Strauss A, Corbin J. Grounded Theory Methodology, An overview. In Denzil, N.K. y Lincoln, Y.S. (1994). Handbook of Qualitative Research. New York: Sage Publications. 1994.

8. Corvalán O, Hawes G. Aplicación del enfoque de competencias en la construcción curricular de la Universidad de Talca, Chile. Revista Iberoamericana de Educación. 2006; 40 (1): 1-17. https://doi.org/10.35362/rie4012528.
9. Frenk J, Chen L, Bhutta ZA, Cohen J, Crisp N, Evans T, et al. Health Professionals for a New Century: Transforming Education to Strengthen Health Systems in an Interdependent World. 2010. https://doi.org/10.1016/ S0140-6736(10)61854-5.

10. Camelo SHH, Angerami ELS. Professional competence: The building of concepts and strategies developed by health services and implications for nursing. Texto e Contexto Enfermagem 2013; 22 (2): 552-60. https://doi. org/10.1590/S0104-07072013000200034

11. Escobar-Castellanos B, Cid-Henriquez P. El cuidado de enfermería y la ética derivados del avance tecnológico en salud. Acta bioethica 2018; 24 (1): 39-46. https://dx.doi. org/10.4067/S1726-569X2018000100039.

12. Dias Sobrinho J. Acreditación de la educación superior en América Latina y el Caribe. En C. Sanyal, y J. Tres (coords.). La educación superior en el mundo 2007: Acreditación para la garantía de la calidad: ¿Qué está en juego? (pp. 282-295). Madrid: Mundiprensa. 2007. 